\title{
Functional ANOVA modelling of pedestrian counts on streets in three European cities
}

\author{
David Bolin $\dagger$ \\ King Abdullah University of Science and Technology, Saudi Arabia \\ Vilhelm Verendel, Meta Berghauser Pont, Ioanna Stavroulaki, Oscar Ivarsson \\ Chalmers University of Technology, Sweden \\ Erik Håkansson \\ University of Gothenburg, Sweden
}

\begin{abstract}
Summary. The relation between pedestrian flows, the structure of the city and the street network is of central interest in urban research. However, studies of this have traditionally been based on small datasets and simplistic statistical methods. Because of a recent large-scale cross-country pedestrian survey, there is now enough data available to study this in greater detail than before, using modern statistical methods. We propose a functional ANOVA model to explain how the pedestrian flow for a street varies over the day based on its density type, describing the nearby buildings, and street type, describing its role in the city's overall street network. The model is formulated and estimated in a Bayesian framework using hour-by-hour pedestrian counts from the three European cities Amsterdam, London, and Stockholm. To assess the predictive power of the model, which could be of interest when building new neighborhoods, it is compared with four common methods from machine learning, including neural networks and random forests. The results indicate that this model works well but that there is room for improvement in capturing the variability in the data, especially between cities.
\end{abstract}

Keywords: Bayesian modeling; count data; pedestrian flows; space syntax.

\section{Introduction}

The theory of natural movement (Hillier et al., 1993) aimed to demonstrate the primacy of characteristics of the street network (i.e., street configuration) over attractions (e.g., land and building uses, such as shops and restaurants, cultural uses etc.) when it comes to the influence on the distribution of pedestrian movement in urban space. Numerous studies have investigated the impact of street configuration (Peponis et al., 1989; Hillier et al., 1987; Hillier and Iida, 2005) and attraction (Peponis et al., 1997; Read, 1999; Berghauser Pont and Marcus, 2015; Ozbil et al., 2011, 2015; Netto et al., 2012; Legeby, 2013; Ståhle et al., 2017) on pedestrian movement. For instance, Berghauser Pont and Marcus (2015) and Ståhle et al. (2017) showed that correlations between diffferent space

$\dagger$ Address for correspondence: David Bolin, King Abdullah University of Science and Technology, Thuwal 23955-6900, Saudi Arabia.

E-mail: david.bolin@kaust.edu.sa 
syntax measures and pedestrian flows vary depending on built density and the morphological characteristics of a neighborhood. Even though different studies of this kind for different regions of the world generally have reported consistent results, there have also been methodological inconsistencies. For example in the kind of empirical data used, the explanatory variables tested, or the statistical methods applied. The datasets have typically been small and seldom been based on data samples that can be said to represent cities as a whole, let alone comprise several cities allowing for comparison. Recently, this has changed with big data availability such as car flows for whole Britain (Serra and Hillier, 2019) and large-scale cross-country pedestrian surveys (Berghauser Pont et al., 2019). They (ibid.) found a significant correlation between characteristics of the urban environment, more specifically built density and street centrality, and the number of people on the street based on a full day pedestrian count survey in several neighbourhoods in three cities. The authors demonstrate that built density explains the intensity of pedestrian flow, while variations in street centrality, or relative position in the system as whole, explain the distribution of this intensity within each area.

Two research questions are addressed in this work. First, whether the same variables, built density and street centrality, that have shown to explain the full day pedestrian counts, can also explain the variation in pedestrian intensities over the day. Second, whether the results can also be used for prediction. It is important to understand how such strategic design variables affect urban life qualities because it informs urban planners how street design and proposed development densities can be used to create areas with a 24/7 economy associated with urban buzz or, on the contrary, create quiet residential areas. The prediction of movement patterns based on design variables is important to evaluate which design alternative performs better in case an urban buzz is aimed for, but it is also highly relevant for, e.g., traffic management and public safety (Zheng et al., 2014) and economic prosperity (Wang et al., 2017). For both explanatory and prediction models, it is important to know whether the results differ across cities and therefore, we include three European capital cities in this study: Amsterdam, London and Stockholm.

Statistically predicting the fluctuations in pedestrian intensities is a more challenging problem than predicting the full day counts, since simple ANOVA analysis similar to that in Berghauser Pont et al. (2019) cannot be used due to the fact that the response for each street now is an integer valued function, representing the number of pedestrians for each hour. We instead propose a generalized linear model that can be thought of as a Bayesian functional ANOVA model (Kaufman and Sain, 2010; Yue et al., 2019) with a negative binomial likelihood. The model uses as explanatory variables, besides density and street characteristics, some additional factors that have earlier been shown to be important in explaining pedestrian movement, such as presence of local markets and proximity to public transport and schools (Stavroulaki et al., 2019). As the goal is not only to explain pedestrian patterns, but also predict them, we compare the proposed functional ANOVA model with common methods from machine learning, such as random forests and artificial neural networks, in terms of predictive power. The reason for this exercise is to see if we could improve on the predictive power by sacrificing the interpretability of the results. Somewhat surprisingly, we find that this is not the case for the methods investigated.

The outline of the paper is as follows. Section 2 contains a detailed description of the data. Section 3 presents the proposed statistical model as well the different machine 
learning methods that are used. Section 4 presents the results of fitting the statistical model to the data, and Section 5 contains the results of a cross-validation comparison where the predictive power of the model is investigated. The article concludes with a discussion in Section 6.

\section{Data description}

In this section, we introduce the dataset that is used in this work. As a part of exploring the data, a web applicationł was developed to provide interactive visualizations of pedestrian movements and street network related features. It also includes plots and summaries from a few regression models that predict total count of pedestrians per street. The application was developed using the $\mathrm{R}$ package shiny (Chang et al., 2019), and several of the figures shown later are produced using the application.

\subsection{Empirical data on pedestrian movement}

Data on pedestrian movement were collected in Amsterdam, London, and Stockholm via tracking anonymized Wi-Fi signals from mobile phones (Berghauser Pont et al., 2019; Stavroulaki et al., 2019). The pedestrian survey was done within a three-week period in October 2017 and included 19 neighborhoods in Stockholm, 18 in Amsterdam, and 16 in London. The detection devices were positioned at every street crossing in the selected areas. The data was processed by street segment (i.e., street between two crossings) and hour. A count was included in each street segment when the wi-fi signal of a unique mobile phone (known as wi-fi probe request) was detected at both its adjacent detection devices (i.e., both street crossings of the segment). The processing included further "noise" removal (such as signals from wi-fi printers), scaling (based on manual counts) and extrapolation (for example, when a device malfunctioned for a brief period). A threshold of $6 \mathrm{~km} /$ hour was used to sort out signals coming from pedestrians and not, for example, from drivers. Each neighborhood was monitored continuously for one workday from $6 \mathrm{AM}$ in the morning to $10 \mathrm{PM}$ in the evening. The dataset includes 219 street segments in Stockholm, 247 in Amsterdam, and 212 in London.

The areas were selected with the objective to cover all building and street types ranging from small alleys to main streets, in neighborhoods which differed in building type, from suburban villa areas of low density to central high-density areas with primarily closed building blocks (see for more details, Section 2.2). The selected neighborhoods included business districts, mixed-use areas, and residential areas of different income level. Because we are interested in the isolated effect of urban types on pedestrian movement, we ensured that no big singular attractors such as train stations, large religious buildings, higher education institutions, cultural buildings, or large shopping malls were located on or near the selected streets (within 500 meter). The main excluding criteria was when the catchment of the attractor extended the neighbourhood it was located. The locations of the measured neighbourhoods as well as some examples of count data from neighbourhoods are shown in Figure 1 and Figure 2. Figure 3 shows an excerpt from the data split up by the types described below.

$\ddagger$ Address for Web Application: http://smogapps.ace.chalmers.se/stepflow 

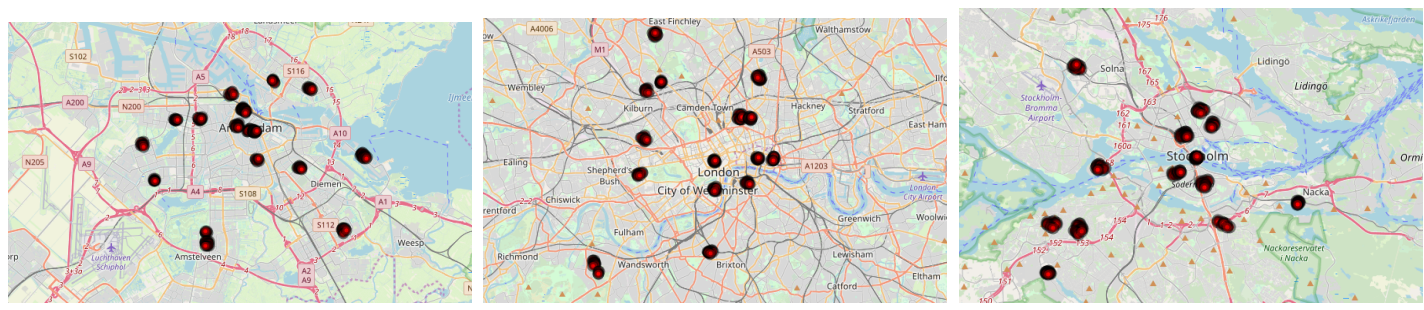

Fig. 1. Neighbourhood locations in Amsterdam (left), London (mid), and Stockholm (right).
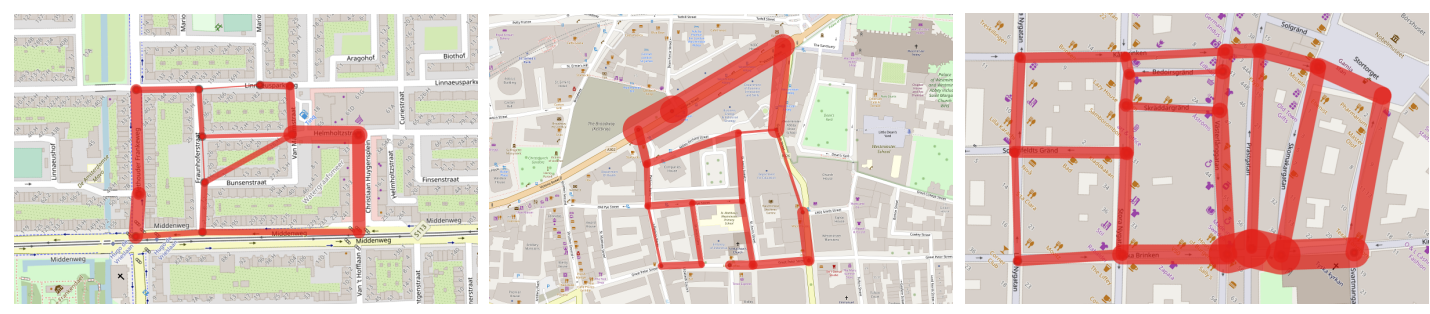

Fig. 2. Examples of neighbourhoods in Amsterdam (left), London (mid), and Stockholm (right). The lines connects the positions of the detection devices and are scaled according to the number of measurements collected. With wider lines refer to higher counts.

\subsection{Density Types and Street Types}

Besides the counts, we have access to a number of covariates describing the streets and neighborhoods these streets are located in. Our focus is on the categorical covariates arising from the typology of Berghauser Pont et al. (2019), which divides the streets into seven different density types (or building types) and four different street types (or centrality types). Each street segment belongs to one density type and one street type. Both are calculated through clustering analysis based on building density metrics for the density types (details are given below) and angular betweenness centrality measured at different scales for street types. K-medoids unsupervised and k-means semi-supervised cluster analysis was used to generate the street and density types respectively; details are described in (Berghauser Pont et al., 2019).

The clustering for density types is based on two measures of density known as floor space index (FSI) and ground space index (GSI). Specifically, these measures are computed in a $500 \mathrm{~m}$ radius around each street, using network distance. Roughly speaking, FSI is the ratio of total floor area to lot area, and GSI is the ratio of built-up area (i.e., footprint of the building) to lot area. Thus, FSI is a measure of the density of total floor area, and GSI is a measure of how large part of the land is taken up by buildings. Note that while GSI always is between zero and one, FSI can in principle take any positive value as buildings can have multiple floors and hence more square footage than the lots they are placed on. Together these two metrics have shown to describe characteristics of the built environment well and allow to distinguish between areas with similar FSI that can be realised with tall buildings, occupying little land or perimeter building blocks that occupy a lot of land and thus can be less tall and still reach similar FSI as high-rise buildings (Berghauser Pont and Haupt, 2010; Steadman, 2013). High-rise developments 

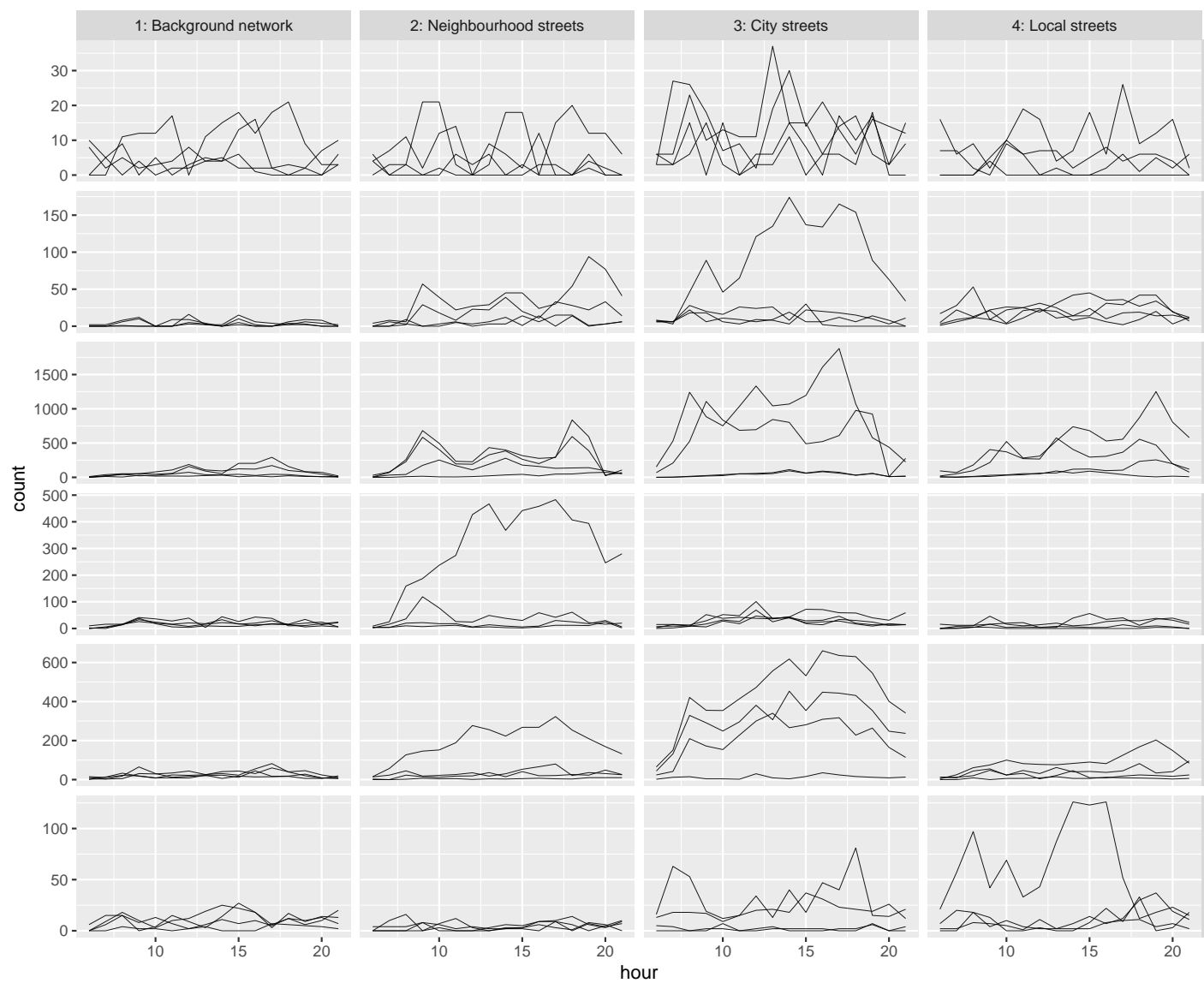

Fig. 3. Counts for four randomly chosen streets in each density-street group.

typically have high FSI and low GSI values, while perimeter blocks can have similar high FSI with lower GSI values.

Seven density types are found in the three cities studied, but because one type is only found in London, no streets were selected for the pedestrian survey in these particular areas. Type 3 (dense mid-rise) is typical in city centres with high FSI and GSI values. The area directly surrounding the city centre typically belongs to type 5 (compact midrise), which has a slightly lower FSI and GSI than type 3. Streets in both these types have been shown to have high pedestrian counts (Berghauser Pont et al., 2019). Type 1 (spacious low-rise) consists to a large extent of villa areas with low FSI and GSI values; type 2 (compact low-rise) represents more compact low-rise buildings and often take the form of row- or townhouses; type 6 (spacious mid-rise) is characterized by spacious configurations of medium high buildings and can be found in many post-war areas following the principals of the functional city. The last density type, type 4 (dense low-rise), represents very compact low-rise building configurations and are found both in industrial areas and historic (i.e., medieval) city centres. An overview of the spatial distribution of density types is shown in Figure 4. 


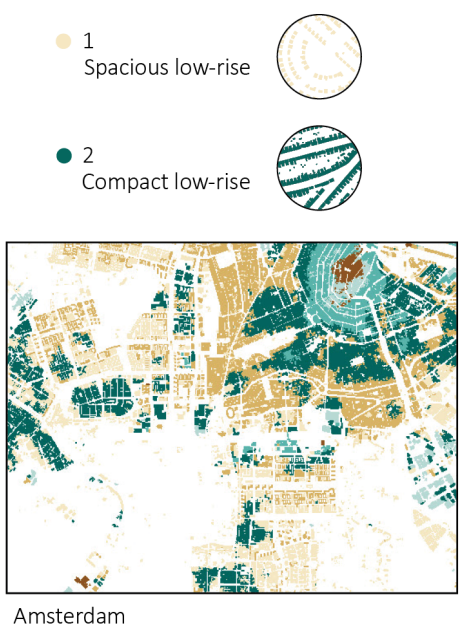

Amsterdam
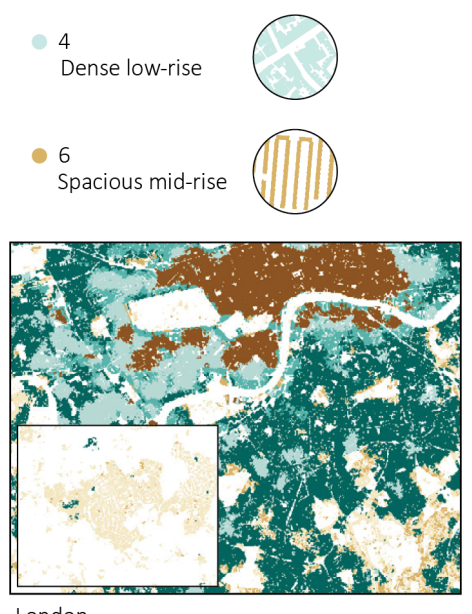

London
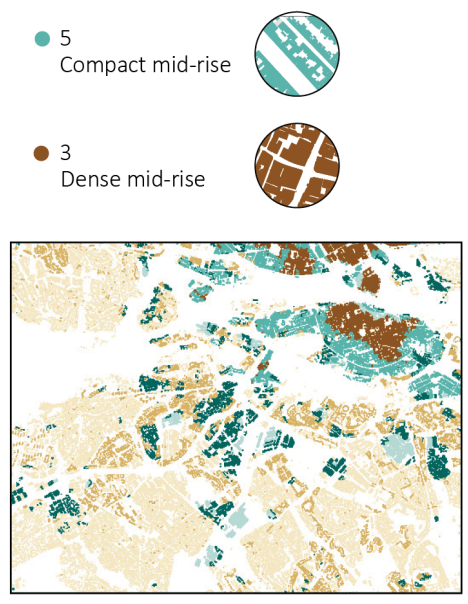

Stockholm

Fig. 4. The spatial distribution of density types in Stockholm, Amsterdam and London. The city cores are located in the upper right corner of each frame. An extra frame has been added for London, to show the distribution at the periphery.

The street types are based on ten angular betweenness centrality measures on different scales (500m until $5 \mathrm{~km}$ with $500 \mathrm{~m}$ intervals) (Hillier et al., 2012). Angular centrality measures calculate the distance between two contiguous street segments by way of measuring their angular deviation. In extension, the shortest path between two segments is the path with the least angular turns in total. Angular betweenness centrality of a street segment is the number of shortest paths between all other segments in the network that pass through it. A street segment with high betweenness is one that falls most frequently on such shortest paths, as for example bridges. On the other end, dead-end streets have zero betweenness centrality since no path passes through them. This analysis can be repeated at several scales capturing the local through movement when a $500 \mathrm{~m}$ radius is used or a more global through movement at the higher radii. The clustering of street centrality resulted in four street types with different profiles or, to use the terms of Peponis et al. (2015), differentiation of scale. The background streets (1) represent the majority of street segments and have low angular betweenness values at all scales; city streets (3) include street segments with increasing angular betweenness centrality at higher scales and thus play a more important role in through movement at the $5 \mathrm{~km}$ scale; neighbourhood streets (2) represent street segments with consistently high betweenness on most scales, but dropping clearly on the lowest and highest scales; these streets have an important function at the in-between neighbourhood level. The fourth type, local streets, includes street segments with high betweenness only on the very local scale, but dropping centrality on all others. Thus, these street segments have an important role in through-movement within neighbourhoods. An overview of the spatial distribution of street types is shown in Figure 5 and the number of streets for each street type and density type is shown in Table 1.

Summarizing the above, the density type is a function of the kind of area a street is lo- 


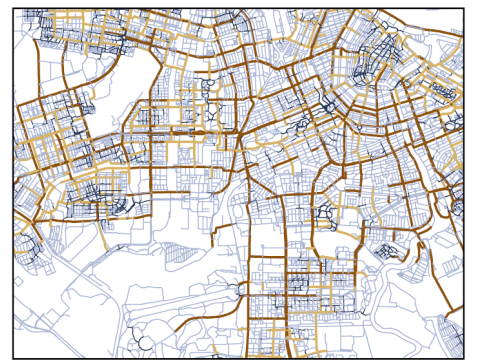

Amsterdam

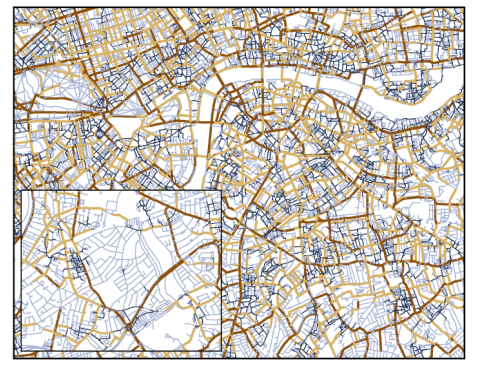

London

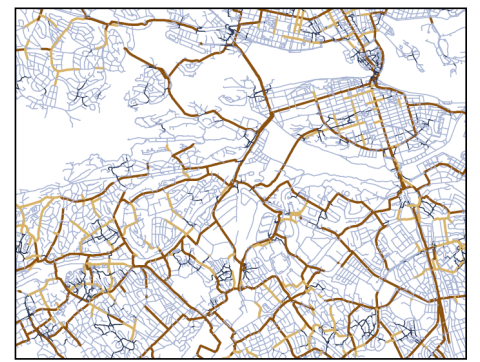

Stockholm

Fig. 5. The spatial distribution of street types in Stockholm, Amsterdam and London. The city cores are located in the upper right corner of each frame. An extra frame has been added for London, to show the distribution at the periphery.

Table 1. The number of streets in each category of street types and density types.

\begin{tabular}{lllllll}
\hline & $\begin{array}{l}\text { 1: Spac. } \\
\text { low-r. }\end{array}$ & $\begin{array}{l}\text { 2: Comp. } \\
\text { low-r. }\end{array}$ & $\begin{array}{l}\text { 3: Dense } \\
\text { mid-r. }\end{array}$ & $\begin{array}{l}\text { 4: Dense } \\
\text { low-r. }\end{array}$ & $\begin{array}{l}\text { 5: Comp. } \\
\text { mid-r. }\end{array}$ & $\begin{array}{l}\text { 6: Spac. } \\
\text { mid-r. }\end{array}$ \\
\cline { 2 - 7 } 1: Background & 60 & 36 & 74 & 32 & 89 & 37 \\
2: Neighborhood & 12 & 16 & 20 & 27 & 18 & 23 \\
3: City & 8 & 18 & 21 & 10 & 22 & 8 \\
4: Local & 23 & 35 & 48 & 10 & 14 & 17 \\
\hline
\end{tabular}

cated in; the street type rather describes what type of street it is. Berghauser Pont et al. (2019) showed that the street and density types have weak, but significant correlations to pedestrian flows in all three cities when including the types separately, but substantial correlations when combining street and density types. In other words, density types explain the intensity of pedestrian flow, while street types explain the distribution of this intensity within the areas. There are two major reasons for using the categorical variables instead of the continuous variables they are built from. The first is interpretability. The interpretation of the continuous variables is somewhat opaque, while the type variables can be interpreted more easily. This is also useful when planning new urban developments, as it is easier to design with and communicate performance of street and density types than the specific values of FSI or angular betweenness at various scales.

\subsection{Attraction variables}

We also include some variables, extracted from the point datasets of Open Street Maps, relating to attractions that are associated with pedestrian movement, albeit their influence can be very specific at entrance and exit times such as in the case of schools or public transport. The attractions included are public transportation nodes (i.e., tram and bus stops), schools (including kindergartens, but excluding higher education) and local markets (i.e., all ground floor retail shops, restaurants and cafes). To capture both the number of individual attractions on the street that could potentially make it a destination point for pedestrian movement, but also the general number of attractions on each 
street's adjacent streets and local context, which could make it a potential thoroughfare between further destinations, we included two measures for each attraction: first, the number of attractions on each segment and second, the number of attractions accessible within walking distance $500 \mathrm{~m}$ from each street segment.

\section{Models and methods}

In this section we describe the proposed statistical model, as well as the machine learning methods that are used for comparison.

\subsection{A functional ANOVA model}

Since our main interest is to use the categorical covariates to explain the variations of pedestrian counts over the day for each street, a functional ANOVA model inspired by the models considered in (Yue et al., 2019) is a reasonable starting point. We model the count for every street and hour as a negative binomial distribution. The average count changes from hour to hour and how it changes is assumed to depend on the density type and street type of the street. Each city is given a separate, time-independent intercept term. In addition to this, we include the attraction variables for schools, local markets, and public transport nodes as covariates that affect the total expected count for the street but not how it changes over time.

Specifically, let $y_{k}(t)$ denote the pedestrian count on street $k$ at time (hour) $t(t=$ $6,7, \ldots, 21)$, which we assume follows a negative binomial distribution,

$$
\mathbf{P}\left(y_{k}(t)=n \mid \eta_{k}, s\right)=\frac{\Gamma(n+s)}{\Gamma(s) \Gamma(n+1)}\left(\frac{s}{e^{\eta_{k}(t)}+s}\right)^{s}\left(\frac{e^{\eta_{k}(t)}}{e^{\eta_{k}(t)}+s}\right)^{n}, \quad n \geq 0 .
$$

Here, the size parameter $s>0$ controls the amount of overdispersion compared to a Poisson distribution; $1 / s$ is the overdispersion and the weak limit as $s \rightarrow \infty$ is the Poisson distribution. While a Poisson distribution is perhaps more natural, an earlier analysis of total counts (Stavroulaki et al., 2019) indicated that the data is overdispersed and we therefore use the negative binomial distribution. Later we will also look at the estimate of $s$ to see whether the negative binomial distribution is necessary.

The other parameter of the negative binomial distribution, $\eta_{k}$, is a time-dependent linear predictor related to the mean of $y_{k}$ through $\eta_{k}(t)=\log \mathrm{E}\left[y_{k}(t)\right]$. Finally, the linear predictor is related to the covariates through

$$
\eta_{k}(t)=X_{k} \phi+\tilde{\mu}(t)+\tilde{\alpha}_{i[k]}(t)+\tilde{\beta}_{j[k]}(t)+\tilde{\gamma}_{i[k], j[k]}(t) .
$$

Here $X_{k}$ is a row-vector of time-independent fixed effects such as schools and local markets, and $\phi$ are the corresponding fixed effects coefficients. Besides the attraction variables described in Section 2.3, the fixed effects include separate intercepts for each city as well as separate intercepts for each combination of street types and density types. A complete list of the fixed effects used in the model is given in Figure 6.

We also have time-dependent, or functional, effects $\tilde{\mu}, \tilde{\alpha}_{i}, \tilde{\beta}_{i}, \tilde{\gamma}_{i, j}$. The first of these, $\tilde{\mu}$, is a baseline time effect, which is the same for all streets. The second $\tilde{\alpha}_{i}, i=1,2,3,4,5,6$ depends on which density type $i[k]$ that street $k$ has. The third, $\tilde{\beta}_{j}, j=1,2,3,4$ instead 
depends on the street type $j[k]$ of street $k$, and finally $\tilde{\gamma}_{i, j}$ is an interaction effect between density type and street type. To ensure identifiability we require that $\tilde{\alpha}_{1}(t)=\tilde{\beta}_{1}(t) \equiv 0$, as well as $\tilde{\gamma}_{1, j}(t) \equiv 0$ for all $j$ and $\tilde{\gamma}_{i, 1}(t) \equiv 0$ for all $i$. We will in the sequel split up the time effects as

$$
\tilde{\mu}(t)=m+\mu(t), \quad \tilde{\alpha}_{i}(t)=a_{i}+\alpha_{i}(t), \quad \tilde{\beta}_{j}(t)=b_{j}+\beta_{j}(t), \quad \tilde{\gamma}_{i, j}(t)=c_{i, j}+\gamma_{i, j}(t),
$$

where $m, a_{i}, b_{j}, c_{i, j}$ are time independent mean levels. The previous identifiability constraint means that $a_{1}=b_{1}=c_{1, j}=c_{i, 1}=0$ and $\alpha_{1}(t)=\beta_{1}(t)=\gamma_{i, 1}(t)=\gamma_{1, j}(t) \equiv 0$. This split is done to more clearly separate changes over time from mean levels in groups, so that we can investigate whether the different types have effects on both the expected total number of pedestrians as well as their distribution over the day.

For the functional effects, we assume second-order random walk priors with the constraint that they should sum to zero for identifiability. That is,

$$
\sum_{t} \mu(t)=\sum_{t} \alpha_{i}(t)=\sum_{t} \beta_{j}(t)=\sum_{t} \gamma_{1, j}(t)=\sum_{t} \gamma_{i, 1}(t)=0, \quad \forall i, j .
$$

A vector $x=\left(x_{1}, \ldots, x_{n}\right)$ is a second order random walk with precision $\kappa>0$ if its second order differences $x_{i}-2 x_{i+1}+x_{i+2}$ are independent and $\mathrm{N}\left(0, \kappa^{-1}\right)$ distributed conditionally on $\kappa$. There are $n-2$ such differences, giving the density $\pi(x \mid \kappa) \propto$ $\kappa^{(n-2) / 2} \exp \left(-\frac{\kappa}{2} x^{t} Q x\right)$, where matrix $Q$ has the form

$$
Q=\left(\begin{array}{ccccccc}
1 & -2 & 1 & & & & \\
-2 & 5 & -4 & 1 & & & \\
1 & -4 & 6 & -4 & 1 & & \\
& \ddots & \ddots & \ddots & \ddots & \ddots & \\
& & 1 & -4 & 6 & -4 & 1 \\
& & & 1 & -4 & 5 & -2 \\
& & & & 1 & -2 & 1
\end{array}\right)
$$

See for example (Rue and Held, 2005, Ch.3) for further details. It is assumed that the different functional effects are independent a priori. Furthermore, we assume that $\mu(t)$ has precision $\kappa_{\mu}$, and that all $\alpha_{i}(t)$ share a single precision parameter $\kappa_{\alpha}$. Similarly all $\beta_{j}(t)$ have precision parameter $\kappa_{\beta}$, and all $\gamma_{i, j}(t)$ have precision parameter $\kappa_{\gamma}$.

The second order random walk priors are reasonable for temporal effects that are not varying too quickly. In an initial analysis, we also tested a model with first-order random walk priors (Rue and Held, 2005, Ch.3) to allow for more quickly varying estimates. This did not improve the model fit.

Finally, it should be noted that we, besides the separate intercepts, assume that all parameters of the model are the same across the three cities. We will later in Section 4.4 also analyse each city separately to check the validity of this assumption.

\subsection{Hyperpriors and estimation}

We fit the model in a Bayesian setting and therefore need to specify hyperpriors for the different parameters in the previous section. These are chosen based on the discussion in 
Yue et al. (2019). Specifically, the non-categorical covariates are normalized by centering and dividing by the standard deviation, and we give the intercept a flat prior $\pi\left(\phi_{0}\right) \propto 1$. The other fixed effects $\phi_{i}, i \neq 0$, including the fixed mean effects $m, a_{i}, b_{j}, c_{i, j}$ for each density and street type, are given vague normal priors $\phi_{i} \sim \mathrm{N}(0,1000)$. The usage of the same priors for all $\phi_{i}$ variables is reasonable as the covariates are normalized.

The precision parameters $\kappa_{\mu}, \kappa_{\alpha}, \kappa_{\beta}, \kappa_{\gamma}$ are given independent and rather flat Gamma priors with shape parameter 1 and rate parameter $5 \cdot 10^{-5}$. Using vague Gamma priors such as these is a common choice for precision parameters of random walk models (See, e.g., Rue and Held, 2005, Ch.4).

The final parameter that needs a prior is the overdispersion parameter $s$. Instead of defining a prior on $s$ directly we let $\theta=\log s$ and give $\theta$ the prior

$$
\pi(\theta)=\frac{7}{\theta^{2}} \frac{\left|\psi^{\prime}\left(\theta^{-1}\right)-\theta\right|}{\sqrt{2 \log \left(\theta^{-1}\right)-2 \psi\left(\theta^{-1}\right)}} \exp \left(-7 \sqrt{2 \log \left(\theta^{-1}\right)-2 \psi\left(\theta^{-1}\right)}\right),
$$

where $\psi$ is the digamma function. This is a penalised complexity prior (Simpson et al., 2017a) described in (Simpson et al., 2017b), which essentially controls the model complexity compared to a Poisson distribution.

Since all model components in the linear predictors $\eta_{k}(t)$ have Gaussian distribution, our model is a special case of a latent Gaussian model. Because of this, we can fit it to data using the integrated nested Laplace approximation (INLA) method of Rue et al. (2009), implemented in the R (R Core Team, 2018) package R-INLA (Rue et al., 2009). The idea behind the INLA method is to use Laplace approximations combined with numerical integration to approximate the posterior distributions of interest. See (Yue et al., 2019) for details about the methodology in the context of functional ANOVA models. The big advantage of INLA compared to using Markov Chain Monte Carlo (MCMC) methods to estimate functional ANOVA models (as, e.g., in Kaufman and Sain, 2010) is computational speed. In addition, INLA has the advantage of being deterministic, which means that there is no need to carefully check for convergence of chains and to do multiple runs as is usually necessary when performing MCMC.

Our choices of hyperpriors are the defaults in R-INLA. Initial tests indicated that the later results are insensitive to the specific values of the parameters of the hyperpriors.

\subsection{Machine learning methods for prediction}

For the goal of assessing the predictive capability of the functional ANOVA model, we also fit other models from machine learning/statistical learning, using the Python package scikit-learn (Pedregosa et al., 2011). Part of the purpose is to see how the ANOVA model that, among other things, allows for a certain level of interpretability, performs compared to models that are more of the "black box" kind. We consider four established methods that are known to facilitate fits of complicated relationships.

The first method is k-nearest neighbor regression (Hastie et al., 2009) which interpolates the data locally by weighting the $k$ closest neighboring data points in the feature space dependent on a chosen distance. The target variable is then taken as a weighted average from nearby points in the training data. Thus, the method has as parameters the number of neighbors $(k)$, the distance metric, and the weighting type. 
Table 2. Machine learning methods and hyper parameter optimization. For each method, all hyper parameter combinations were explored. The naming convention follows the implementations in scikit-learn, which was used to implement and evaluate the methods.

\begin{tabular}{lll}
\hline Method & Hyper parameters & Values tested \\
\hline \multirow{2}{*}{ K nearest neighbors } & k: number of neighbors & $1, \ldots, 20$ \\
& distance metric & L1, L2 \\
& weights: influence of neighbors & uniform, distance \\
\hline Random forest & trees & $50,100,200$ \\
& min number of samples, splitting nodes & $1,2,3,5$ \\
& min number of samples, leaf nodes & $1,2,3,5$ \\
& max depth of a tree & $3,5, \infty$ \\
\hline Gradient boosting & trees & $50,100,200$ \\
trees & min number of samples, splitting nodes & $1,2,3,5$ \\
& min number of samples, leaf nodes & $1,2,3,5$ \\
& max depth of a tree & $3,5, \infty$ \\
& validation fraction & $0.1,0.2,0.3$ \\
\hline Artificial neural & $\alpha:$ L2 regularization & $10^{-4}, 10^{-3}, 10^{-2}, 10^{-1}$ \\
network & hidden layers & $1,2,3$ \\
& hidden layer sizes & $20,50,100,200$ \\
\hline
\end{tabular}

The second method is random forest regression (Ho, 1995) which includes multiple decision trees that contribute to predict the data. The decision trees are fit by randomly sub-sampling data when training each decision tree, to provide variance, and then the outcome from the different trees is averaged into a prediction to avoid over-fitting or bias.

The third method is gradient boosting trees (Friedman, 2001), which can be thought of as another generalization of the decision tree concept. Instead of fitting separate decision trees, the trees are fit sequentially to the data, taking into account information from the previously trained decision trees: Given a population of trees, new trees are fit to the residuals of how the previous trees predict a fraction of data used to validate the previous model (Hastie et al., 2009).

Finally, we also train a few feed-forward artificial neural networks (Hinton, 1990), where the number of hidden neuron layers in the model as well as hidden layer sizes are varied. We attempt to prevent overfitting by varying both the network structure as well as a regularization parameter for weight decay for the trained networks.

The hyperparameters that need to be chosen for each method are summarized in Table 2. For each method, we optimize the hyper parameters by testing the method with different values of the parameters (the different values tested are also given in the table) on the available training data before evaluating the performance on separately held out data as described in Section 5.

\section{Model fit for the functional ANOVA model}

In this section we describe and discuss the functional ANOVA model fit. We start considering the the overall mean levels (the fixed effects), then the structure over time 


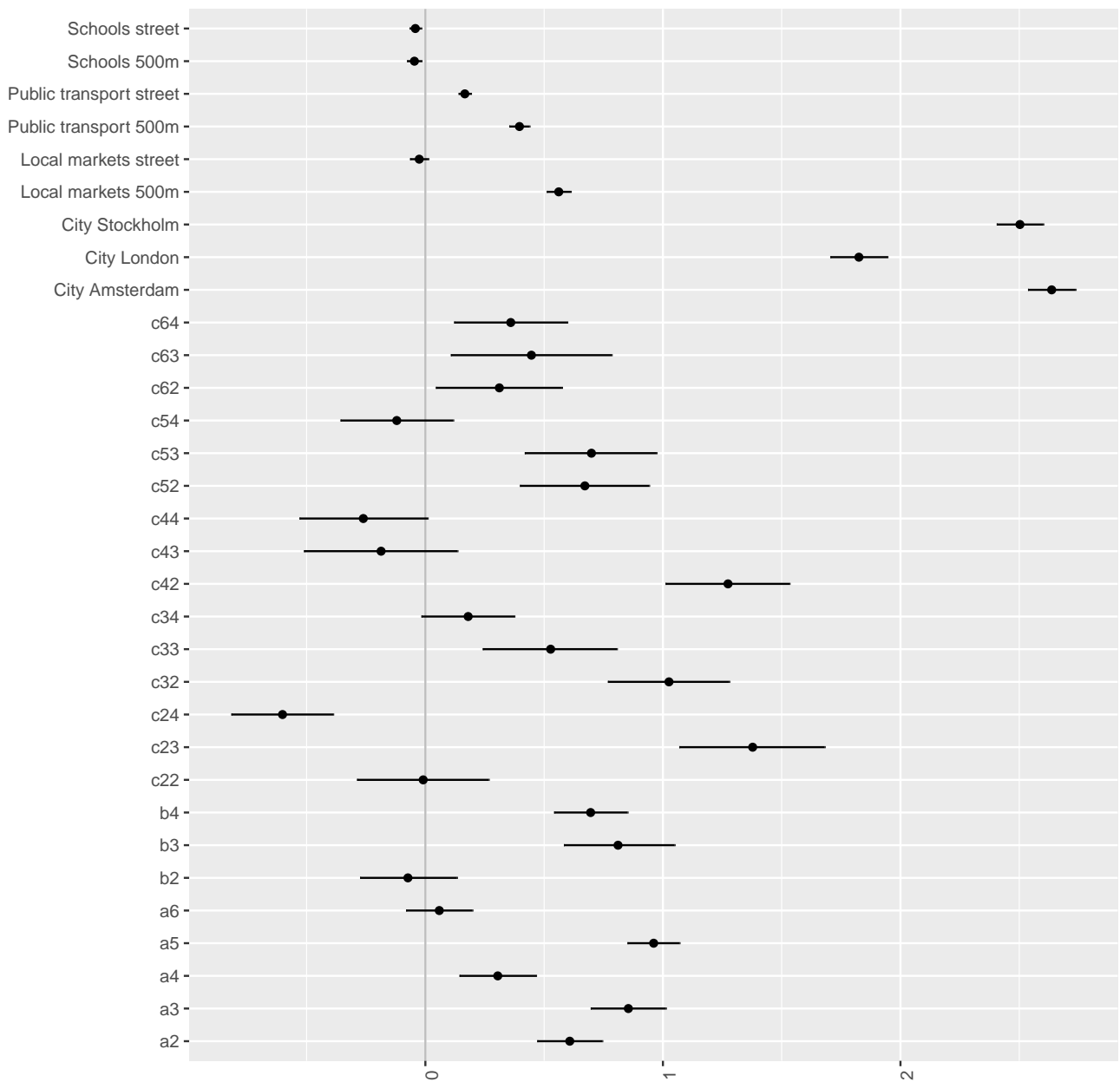

Fig. 6. Fixed effects estimates. Points show posterior means and lines $95 \%$ credible intervals. Here $a, b$, and $c$ denote the effects for density types, street types, and interactions, respectively.

(the functional effects), and finally the general model fit as well as a city-specific analysis.

\subsection{Fixed effects}

The fixed effects estimates are shown in Figure 6. We see that there is a rather clear difference between the cities. The expected pedestrian counts for Amsterdam is largest given that all other covariates are at zero, followed by Stockholm, and then London. However, this might be an effect of the specific streets that were measured in each city. For instance, in Amsterdam less neighborhoods of density type 3 (dense mid-rise) were selected, because most of the city centre (where we get the higher counts) was of density type 4 (dense low-rise) or 5 (compact mid-rise).

Regarding the attraction covariates, the schools seem to have small effect, while the local markets and public transport stops have larger effects. The street-level local markets 
effect is not significant though. A neighbourhood with more retail and food services is thus increasing the number of pedestrians, while this is not the case when these are found on the same street where the observation is made. One could also say that the local market needs to be larger than one street to effect pedestrian movement patterns.

Overall the density fixed effects estimates (the $a_{i}$ parameters) seem natural considering what the types represent; it is no surprise that the typical city centre types, dense midrise (3) and compact mid-rise (5), with the highest FSI and GSI values have clearly larger estimates $\left(a_{3}\right.$ and $\left.a_{5}\right)$ than the other types. Type 6 (spacious mid-rise) has the lowest estimate $\left(a_{6}\right)$, followed by types 4 (dense low-rise) and 2 (compact low-rise). Looking back at the descriptions of the types this is natural, with these types roughly corresponding to suburban and industrial areas where not so much pedestrian traffic is to be expected. It is noteworthy that type 6 (spacious mid-rise) with a relatively high FSI has such low pedestrian counts, showing the importance of other variables than the most often referred density metric FSI. The second variable used for clustering, GSI, is important as well which confirms earlier findings by Berghauser Pont et al. (2019).

One can also note that for all density types the effect estimates $\left(a_{2}\right.$ through $\left.a_{6}\right)$ are positive. Due to the way the model is specified, type 1 (spacious low-rise) is taken as a baseline, with $a_{1}=0$ by definition. This type is characterized by villa developments which have very low pedestrian counts and the all-positive estimates simply mean that all other types of areas have larger pedestrian counts than the villa areas. Similarly, street type 1 which consists of background streets is taken as a baseline so that $b_{1}=0$, and thus the positive estimates for city streets $\left(b_{3}\right)$ and local streets $\left(b_{4}\right)$ mean that these have larger pedestrian counts than the background streets and neighborhood streets $\left(b_{2}\right)$.

For the interaction variables $c_{i j}$ one can clearly see that there are significant interaction effects between the density and street types. In density type 6 (spacious mid-rise) with low expected counts, there is no difference between the interactions with street types, while in all other types we see the lowest counts in the local street and the highest in the neighbourhood street, which confirms the theory of the importance of overlapping scales (Peponis et al., 2015).

Concluding, we can say that the combination of density and street type is decisive for the understanding of pedestrian movement, which confirms earlier findings by Berghauser Pont et al. (2019), but the results presented here add important details about the specific interaction within each density type. Overall, the patterns show lowest counts in the background and local streets and highest levels in the neighbourhood streets.

\subsection{Functional effects}

The next point to discuss is the time effects. R-INLA directly provides pointwise credible intervals for the estimated effects, but to be able to check for significance of the functional effects we also compute simultaneous credible intervals using the excursion method (Bolin and Lindgren, 2015; Bolin et al., 2015) implemented in the R package excursions (Bolin and Lindgren, 2018). Since density/street type 1 is taken as baseline, we only display the effects for the other types.

Figure 7 shows the baseline effect $\mu$. For this effect we see that there are two peaks, one in the morning and one in the evening. We also see that the effect is clearly significant. Figure 8 shows the time effects $\alpha_{i}$ for each density type. With the exception of type 6 


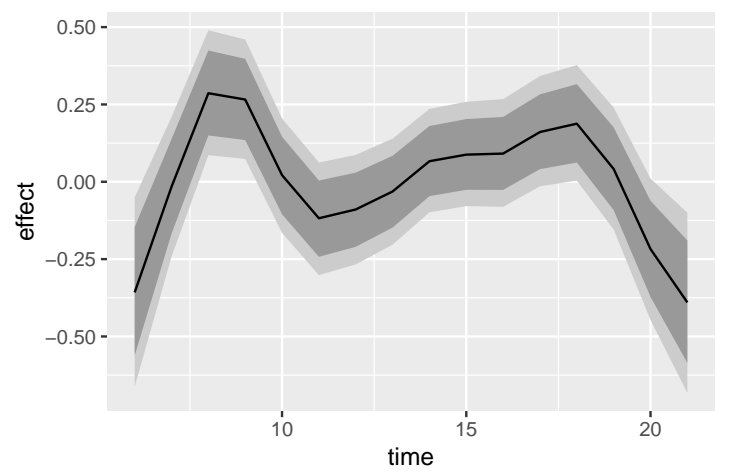

Fig. 7. Temporal baseline effect. Posterior mean (black) and 95\% pointwise (darker) and simultaneous (lighter) credible intervals.

(spacious mid-rise), these are all clearly negative in the early morning. Since type 1 (spacious low-rise) is taken as baseline, this means that the other types will have lower average pedestrian counts in the morning compared to type 1 . Looking at the credible intervals, only the one for type 6 contains the zero function, and hence the other density types seem to have a real effect on the counts over time.

The time effects $\beta_{j}$ of each street type are shown in Figure 9. Unlike the baseline and density type effects, these have credible intervals containing zero, meaning that there might plausibly be no real time effect of the street type. It might therefore not be worth it to include a time effect for the street types. However, since the $b_{j}$ variables are clearly non-zero it is helpful to include a fixed effect for the street types. This is discussed further below when comparing the models. Finally, Figure 10 shows the estimated temporal interaction effects. As for the street type effects, none of these are significant.

\subsection{Additional model checks}

As a sanity check, we compare the actual time effect curves we get for each type combination when we combine the $\mu-, \alpha$-, and $\beta$-effects with the observed data in Figure 11. This figure compares the average observed counts with the effects for each type combination. In order to be able to compare the combined time effects with the observed counts, we have normalized the counts for each street by the part of the mean value that is constant over time, that is, we consider

$$
e_{k}(t)=Y_{k}(t) \exp \left(-X_{k} \phi-a_{i[k]}-b_{j[k]}-c_{i[k], j[k]}\right) .
$$

In the figure, the estimated time profile for each density and street type pair is shown together with the average of the $e_{k}(t)$ values for that case. Empirical $5 \%$ and $95 \%$ quantiles of the $e_{k}(t)$ values are also shown. One can note that the model fits the data reasonably well, but that there also is quite a large variation of the observed counts in some classes. Thus, the sizes of the fluctuations over the day are in most cases small compared to the within-group variability.

It is possible to give reasoning behind some of the behaviours observed in the figure. For example, take group 4-1 which represent local streets in the density type spacious 

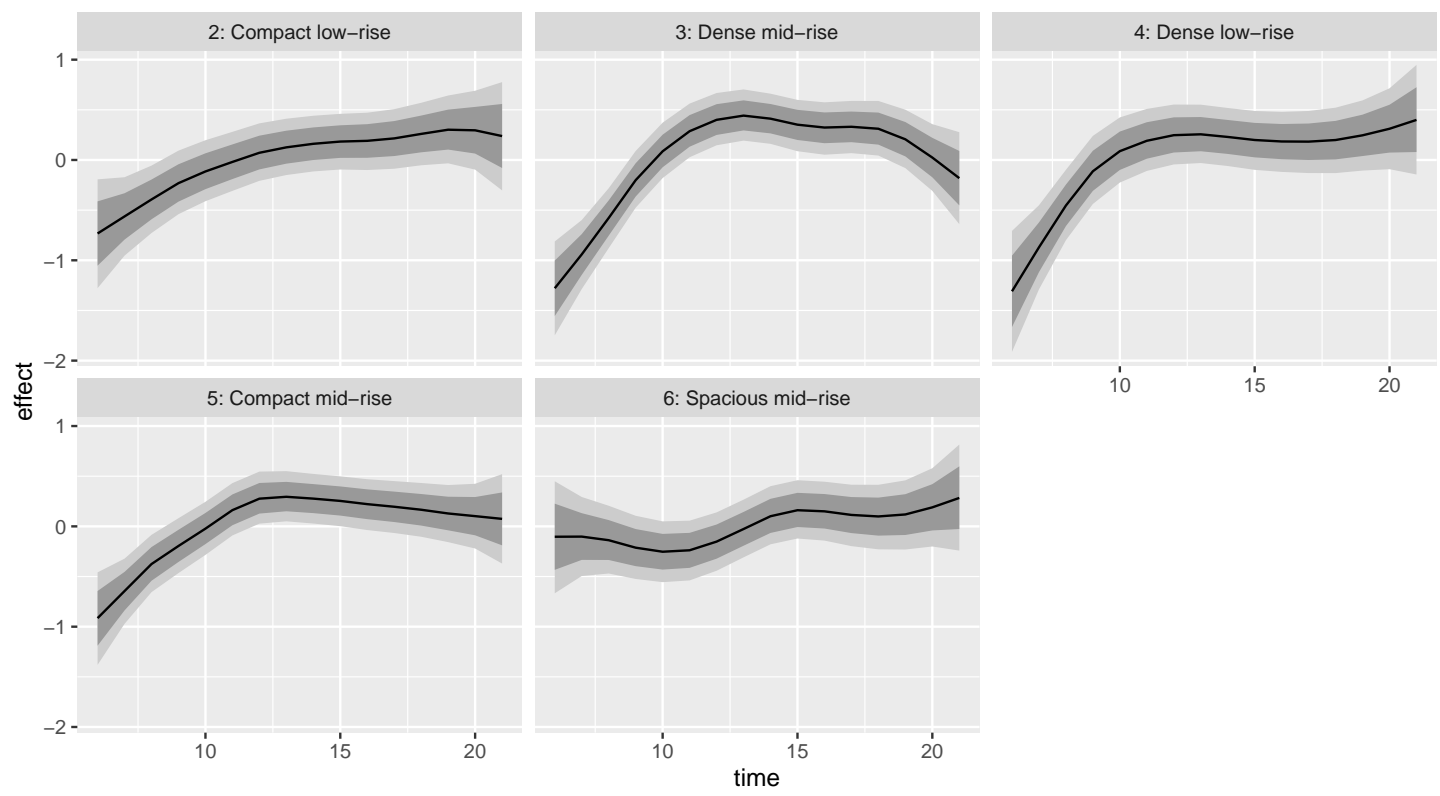

Fig. 8. Time functions for different density types. Solid lines are posterior means, shaded regions $95 \%$ pointwise (darker) and simultaneous (lighter) credible intervals.

low-rise dominated by villa development. This effect has three peaks, one in the morning when people leave for work and one in the evening when people travel home from work, and in between it is more quiet with a subtle peak at lunch. The other streets in this density type show similar patterns, albeit with lower max values. In more dense and compact areas, i.e., density type 3 (dense mid-rise) and 5 (compact mid-rise), there is a stronger lunch peak and the number of people increases towards the early evening. One could speculate that people go out to eat and shop in these areas that in general have more other economic activities.

A natural question is whether the negative binomial likelihood really is needed for the data. To answer this, we compute a $95 \%$ credible interval for the overdispersion (that is, $1 / s$ where $s$ is the size parameter). This interval is $(1.51,1.6)$ which means that the overdispersion is clearly nonzero. Thus the negative binomial distribution (or some other overdispersion adjustment) is necessary, and Poisson regression is not appropriate for this data. Hence we can not simplify the model and use a Poisson regression without undermining the integrity of the model.

As a final simple model check, we use the probability integral transform, or PIT. For an observation $y_{k}$ the PIT value is calculated as $\mathrm{PIT}_{k}=\mathbf{P}\left(Y_{k} \leq y_{k} \mid y_{-k}\right)$, where $Y_{k}$ is distributed according to $\pi\left(y_{k} \mid y_{-k}\right)$, i.e., the predictive distribution of observation $k$ given all other observations. If the model is appropriate for the data then this predictive distribution is really the 'true' distribution for $y_{k}$, and $\mathrm{PIT}_{k}$ should then be uniformly distributed if $Y_{k}$ is a continuous random variable. One can therefore asses the model by for example plotting sorted PIT values against the corresponding uniform quantiles.

An issue with this in our case is that we have discere data, so one cannot expect to 

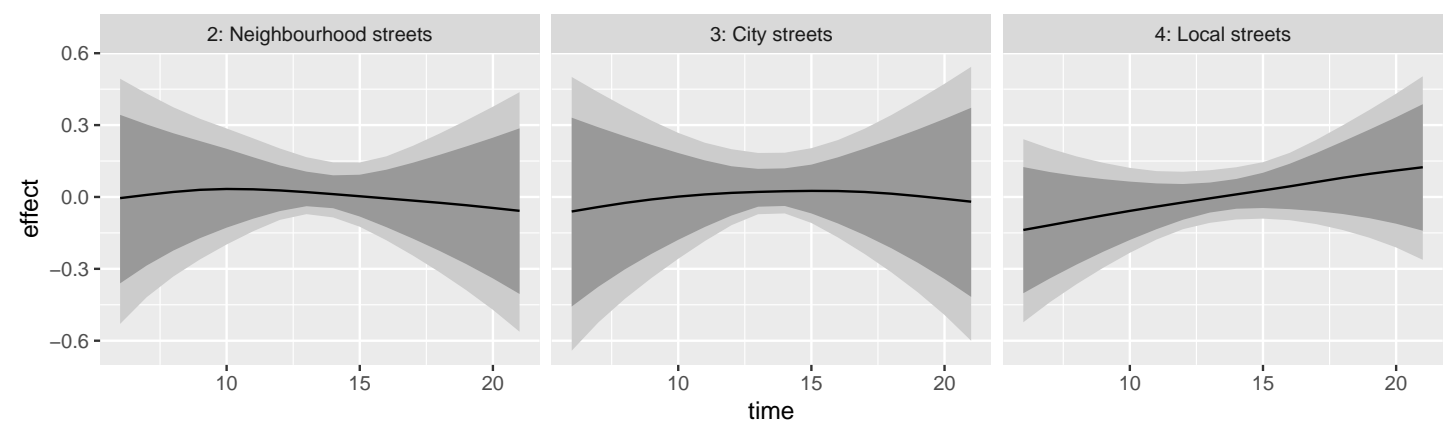

Fig. 9. Time effects for different street types. Solid lines are posterior means, shaded regions $95 \%$ pointwise (darker) and simultaneous (lighter) credible intervals.

get exact uniformity. To handle this we create simulated PIT values, and compare these to the observed ones. This is done by sampling new data from the posterior, refitting the model to this new data and computing the PIT values. The results are shown in Figure 12, where the sorted PIT values are compared to uniform quantiles, with PIT on 20 simulated datasets in grey. The PIT values of the fit do not seem to be uniformly distributed. However, they do not look so different from the simulated values which do not look uniform either. Thus, the model seems to fit the data fairly well.

\subsection{Analysing the three cities separately}

We have assumed that the effect of the different covariates is constant between different cities. However, a natural question is whether there are city-dependent mechanisms that alters the effects of the covariates. As an initial test of this, we fitted the functional ANOVA model without interaction effects (i.e., a model with $\gamma_{i, j} \equiv 0$ ) to all data, but also to the data from Amsterdam and London separately (Stockholm is not included due to the lack of density type 4 streets). Figure 13 shows the density type effects from these models. We can see that there is a rather clear difference between the effect for Amsterdam and London by looking at types 4 (dense low-rise) and 5 (compact mid-rise). Type 4 areas are representing rather different kind of areas across the three cities, so we are not so surprised by the variation in prediction. However, we expected less variation in type 5 areas that are the most urban areas across cities and very homogeneous in their spatial organisation. Our hypothesis is that other variables than those included in the model might play a more important role in these areas, such as the type of economic activities that are present in the areas. High dense areas with more restaurants will have another movement pattern during the day than areas with more shops. The Amsterdam and London estimates also differ from the estimates when using all cities, both in uncertainty (which is to be expected) and shape. Similar behaviour can be observed for the baseline and street effects, though we do not show them here. This is an indication that there are differences between the cities that are not captured by the model. In the light of this, a natural extension of the model to investigate is one where we let the time effects vary by city as well. However, the inclusion of city-by-city variation in this manner impedes the generalisability of the model to cities not in the dataset so 

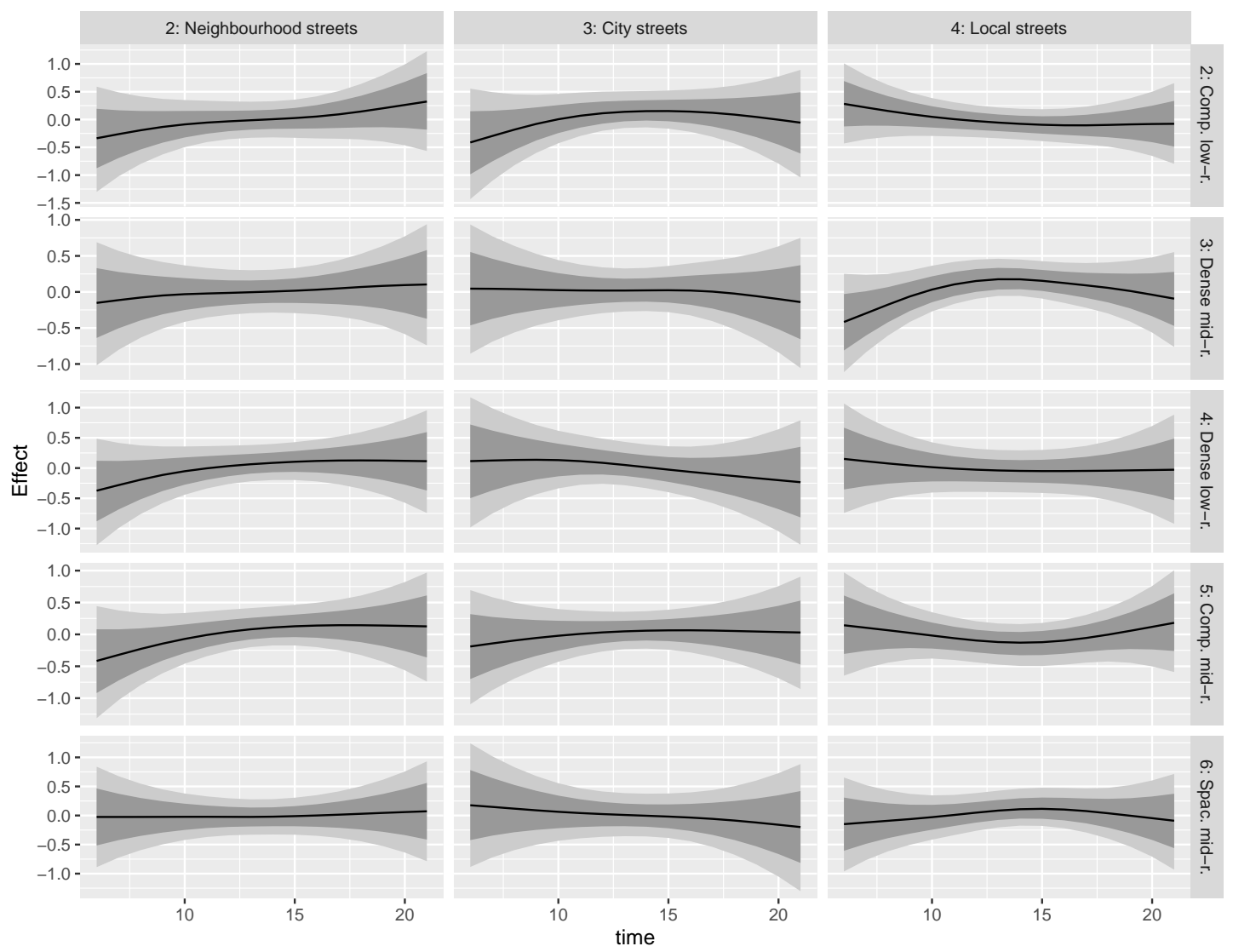

Fig. 10. The estimated interaction effects $\gamma_{i j}$. Solid lines are posterior means, shaded regions $95 \%$ pointwise (darker) and simultaneous (lighter) credible intervals.

if this is to be done one has to make sure that this is not at odds with the modelling goals. Nevertheless, investigations of this type can provide important information when comparing the behaviour of pedestrian flows in different cities.

\section{Cross-validation model comparisons}

Since one potential use of a model for pedestrian flows is prediction, we now compare the functional ANOVA model with various simplified versions of the model as well as the machine learning methods for prediction described earlier.

The simplified versions we test are: A model without temporal interaction effects $\left(\gamma_{i, j} \equiv 0 ;\right.$ ANOVA no interaction); a model without temporal interaction effects and without temporal street type effects $\left(\gamma_{i, j}=\beta_{j}=0 ; A N O V A\right.$ density); a model with only $\mu(t)$ as temporal effect (ANOVA only main); and a model without any temporal effects (i.e., a negative binomial regression model; ANOVA no temporal effects). For all these models, we include all fixed effects, including the type variables and interactions for the average counts, shown in Figure 6. 

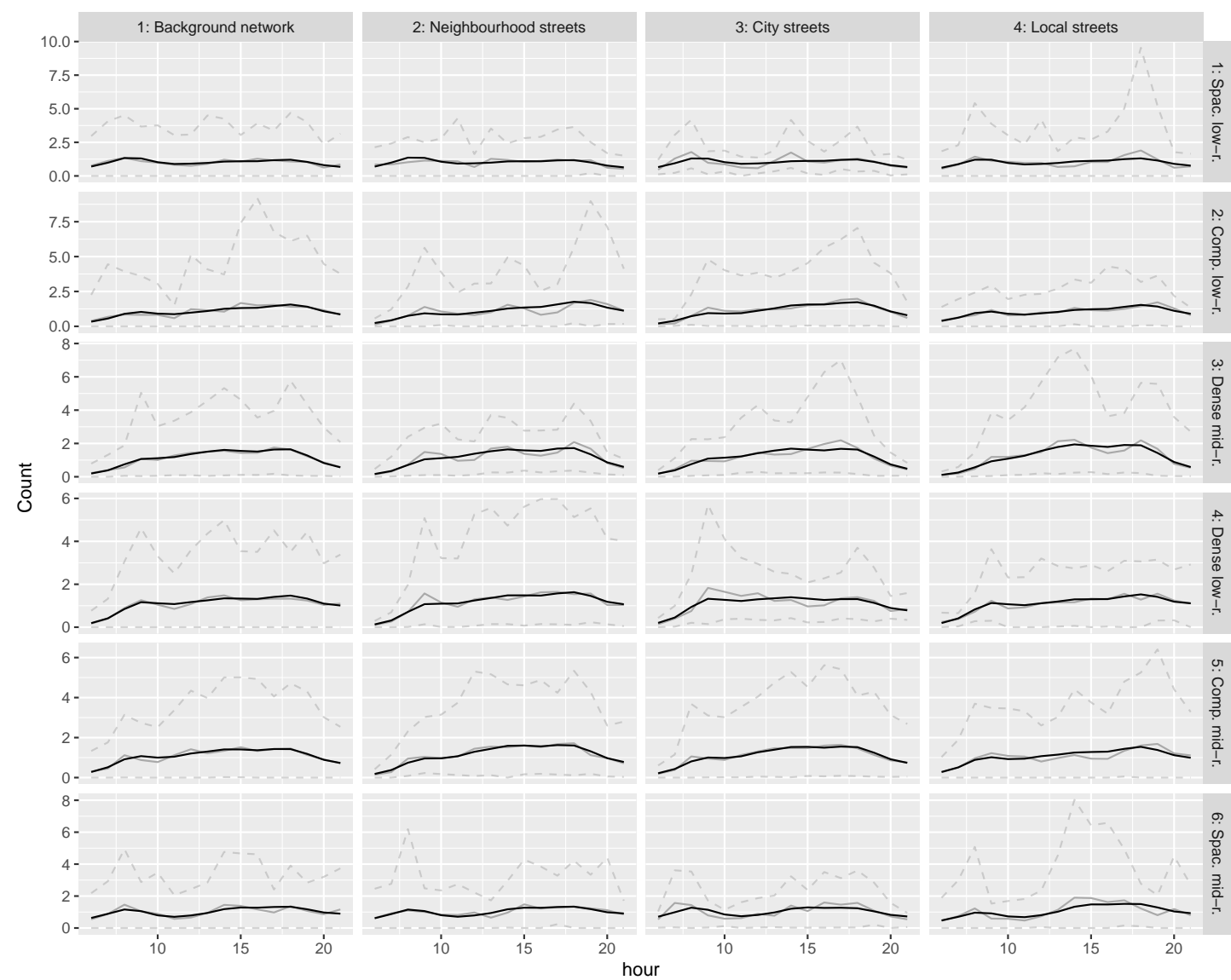

Fig. 11. Normalized average counts (gray, solid) and pointwise $90 \%$ confidence bands (dashed) as well as the expected temporal variation according to the ANOVA model (black), split by density type (rows) and street type (columns).

We test the predictive performance in a cross-validation scenario where the models are fitted to all data from $90 \%$ of the streets chosen at random, and the predictive performance is measured on the held-out $10 \%$ streets. This is repeated 10 times, each with a new random selection for the training data.

To measure the predictive performance, we use mean absolute errors (MAE) and root mean squared errors (RMSE) based on using the posterior mean as predictor for the ANOVA models. To take the accuracy of the prediction uncertainty into account, we also use averages of proper scoring rules to rank the predictive performance. A popular alternative is the continuous ranked probability score (CRPS), which for an observation $y$ and a predictive distribution $\mathbb{P}$ is defined as $\operatorname{CRPS}(\mathbb{P}, y)=\mathrm{E}_{\mathbb{P}}|X-y|-\frac{1}{2} \mathrm{E}_{\mathbb{P}, \mathbb{P}}\left|X-X^{\prime}\right|$. Here $\mathbb{E}_{\mathbb{P}}|X-y|$ denotes the expected value assuming that $X$ has distribution $\mathbb{P}$, and $\mathrm{E}_{\mathbb{P}, \mathbb{P}}\left|X-X^{\prime}\right|$ denotes the expected value assuming that $X$ and $X^{\prime}$ are independent and distributed according to $\mathbb{P}$ (Gneiting and Raftery, 2007). However, this score suffers from giving more weight to parts of the predictive distribution where there is more variability 


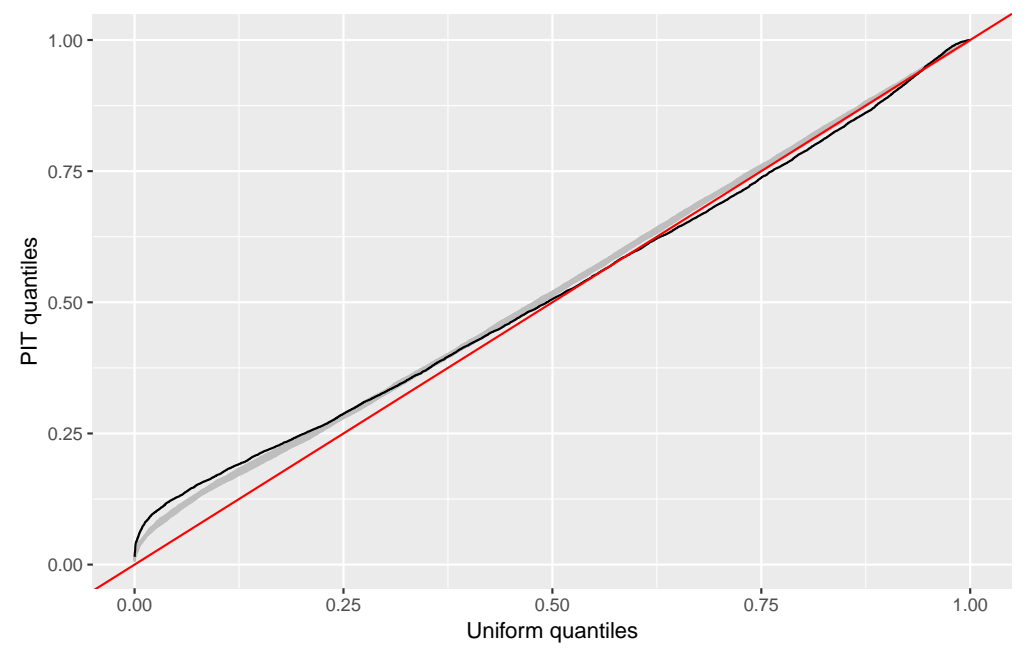

Fig. 12. Sorted PIT values versus uniform quantiles (black); 20 simulated runs in grey.

(Bolin and Wallin, 2020). Since the variability of the negative binomial distribution scales with the mean this will give more weight to streets with large counts which is not appropriate as the goal is to get good results for all kinds of streets. As alternatives, we instead use the Dawid-Sebastiani score

$$
\operatorname{DS}(\mathbb{P}, y)=\frac{\left(\mathrm{E}_{\mathbb{P}}(X)-y\right)^{2}}{\bigvee_{\mathbb{P}}(X)}+\frac{1}{2} \log \bigvee_{\mathbb{P}}(X),
$$

where $\mathrm{V}_{\mathbb{P}}(X)$ denotes the variance of the distribution $\mathbb{P}$, as well as the scaled CRPS proposed in (Bolin and Wallin, 2020),

$$
\operatorname{SCRPS}(\mathbb{P}, y)=\frac{\mathrm{E}_{\mathbb{P}}|X-y|}{\mathrm{E}_{\mathbb{P}, \mathbb{P}}\left|X-X^{\prime}\right|}+\frac{1}{2} \log \mathrm{E}_{\mathbb{P}, \mathbb{P}}\left|X-X^{\prime}\right| .
$$

These two scores are both locally scale invariant and therefore more appropriate for evaluating the predictive performance in our case (Bolin and Wallin, 2020). The expectations were approximated using Monte Carlo integration by simulating from the relevant posterior distributions. The results are shown in Table 3. The scores are not defined for the machine learning methods since they only provide point estimates.

The model without temporal effects where we assume that the number of pedestrians is constant over the day performs worst among the five tested statistical models. The ANOVA only main model adds a single functional effect that is shared among all streets, meaning that every street is assumed to have the same fluctuations over the day. This model performs better, and in fact has the best results in terms of MAE and RMSE. In terms of SCRPS, the best results are found for the ANOVA density model, where we have separate functional effects for each density type, but none for the street types. However, the SCRPS results (and the DS results) are almost equivalent to the model including separate functional effects for each street type, but no interactions. The full ANOVA model where these interaction effects are added to have full control over the 

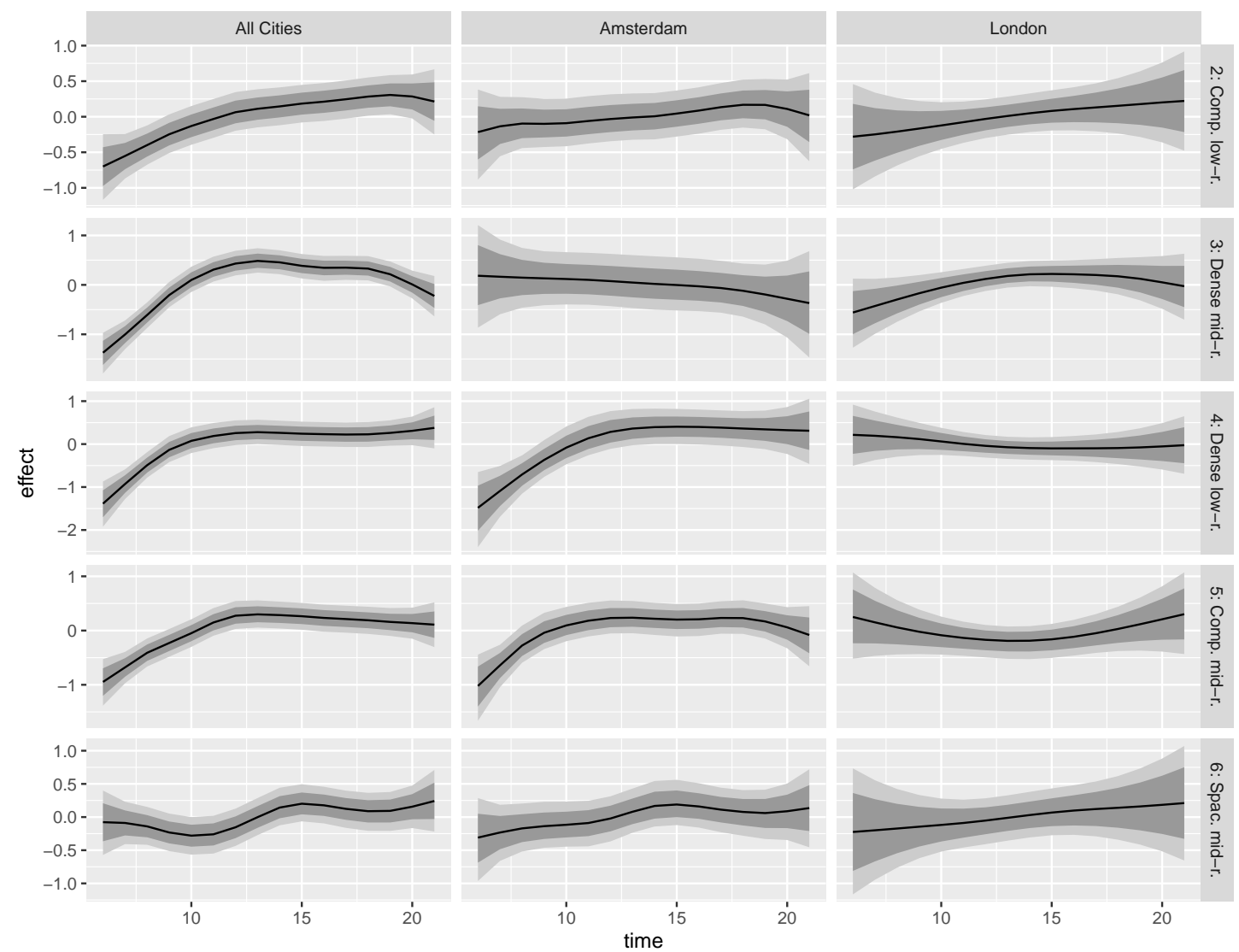

Fig. 13. Density type effects $\left(\alpha_{i}\right)$ for the functional ANOVA model without temporal interaction effects compared to models fitted separately to Amsterdam and London. Lines are posterior means, shaded regions are 95\% pointwise (dark grey) and simultaneous (light grey) credible intervals.

fluctuations for each street type - density type pair also performs similarly in terms of SCRPS, but since the much larger number of functional effects in this model does not improve the results it is likely better to use the simpler models.

We can thus conclude that the street type is not decisive for the fluctuations of pedestrian flows, while density types are. It is worth noting that both street types and the interaction between density and street types are of high importance for the total expected count (see Section 4.1). Hence, density types can explain daily patterns of urban life, while both street and density type are important for the prediction of the total number of people walking the street during a full day.

It should be noted that RMSE and MAE also have the problem that values with high expected counts will be more important. This means that there is a large variability among the results for the different cross validation folds (see Figure 14 for an illustration). Nevertheless, it is interesting to note that the statistical models have better or equal predictive performance than the machine learning models in terms of MAE and RMSE. 
Table 3. Average scores for cross-validation based on 10 random train-test splits. The same splits were used for all models. MAE and RMSE are computed using posterior means as predictions.

\begin{tabular}{lllll}
\hline Model & SCRPS & DS & MAE & RMSE \\
\hline ANOVA full & 3.459 & 5.460 & 44.79 & 115.2 \\
ANOVA no interaction & 3.458 & $\mathbf{5 . 2 2 1}$ & 45.12 & 117.8 \\
ANOVA density & $\mathbf{3 . 4 5 5}$ & 5.266 & 44.99 & 116.6 \\
ANOVA only main & 3.474 & 5.354 & $\mathbf{4 3 . 6 1}$ & $\mathbf{1 0 5 . 2}$ \\
ANOVA no temporal effects & 3.520 & 5.546 & 50.05 & 124.8 \\
K-nearest neighbor & - & - & 43.73 & 116.1 \\
Random Forest & - & - & 49.41 & 142.9 \\
Gradient Boosting Trees & - & - & 49.06 & 133.7 \\
Artificial neural network & - & - & 47.91 & 122.6 \\
\hline
\end{tabular}

Further, the more simple machine learning method (k-nearest neighbor) performs better than the more advanced ones. A likely reason for this is that more data is needed to get improved results with the more advanced methods.

\section{Discussion}

We have proposed and compared several models for how pedestrian counts vary throughout the day, with covariates for both temporal, city-specific, and effects of the built environment, namely built density and street network centrality. We find that for the total pedestrian counts in the observed streets, density and street types as well as their interaction play an important role. For the attraction covariates, the schools seem to have very small effect, while the local markets and public transport stops have larger effects. Further, we find that the sizes of the fluctuations over the day are in most cases small compared to the within-group variability, but that the city effect is substantial. For the temporal fluctuations during the day, the density type has a significant effect, but we did not find a real time effect of the street type. That the fluctuations of pedestrian flows during the day seem to depend mostly on density, while the street characteristics seemed insignificant was also noted by (Berghauser Pont et al., 2019) by visual inspection of average profiles for each group. Thus our formal tests confirm their findings and in addition show that there is no significant interaction effect for the temporal profiles.

For planning and design practice, these results may prove highly significant, as they allow urban planners and designers to propose street types and densities with an understanding of how this will affect urban processes with pedestrian movement patterns as a vital intermediary process. Further, the results provide insights into the importance of the relationship between street and building types in designing areas with different qualities, whether aiming for urban buzz or quiet residential areas. The differences found between the cities highlight at the same time that one cannot generalize the findings to cities not in the dataset, especially not for the neighbourhoods with highest density (type 5) but this is something that needs to be studied in the future in more detail.

The dataset has a large number of observations from a smaller number of streets in three cities, and we find the following patterns when comparing how models fit predict 

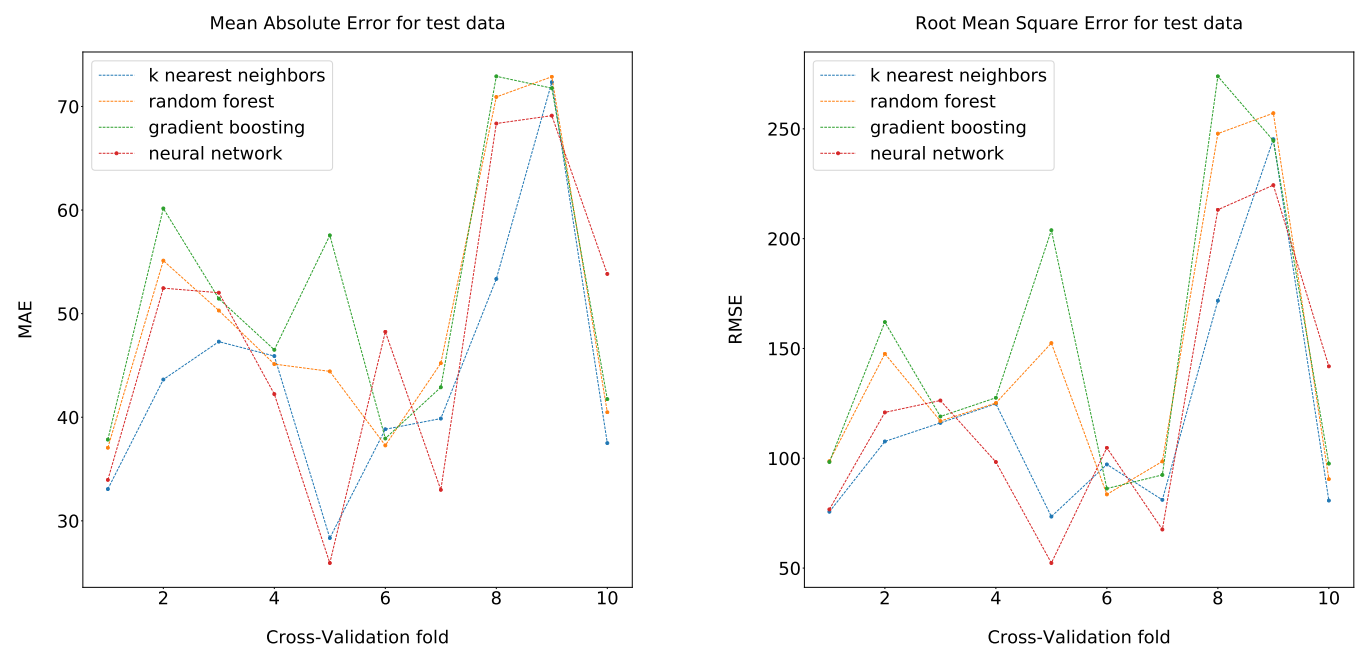

Fig. 14. Comparison of machine learning methods across the same train-test splits used for the other models. MAE and RMSE were computed for prediction on the test data.

the data. First, it is fair to say that for this data and for the prediction task, the functional ANOVA methods work best among the investigated methods. Despite that the machine learning methods can predict counts with similar mean average errors, we find that they are more sensitive to outliers (larger RMSE in Table 3). The results from the cross-validation indicate that the prediction works relatively similar for many of the streets. This shows a clear advantage of the functional regression method, paired with a random walk prior such that it smoothens out the prediction of the models when it comes to the temporal effect; this makes them less sensitive to outlier predictions. Second, we note that for the prediction task, the simpler models work better. This could suggest that we observe over-fitting and that we would need more data. Another possibility to improve the predictions could be to have a more fine-grained taxonomy of streets. See, e.g., Figure 3 which shows that city streets seem to have groupings of streets with quite different levels. Another possibility would be to add higher-order interaction effects to the model. That this is an unbalanced data set in terms of the target variable, where some streets have really high counts, is also seen in the cross-validation (Figure 14). Our interpretation is that more data about streets with really high counts could be valuable for future research.

The assumption that the effects of the attraction variables are constant in time is questionable for some attractions such as schools. The models could be extended with time varying attraction effects; however, initial tests (not reported here) indicate that a time varying school effect only provide a minor improvement to model fit.

A final question for future research is how spatial dependence can be included in the models. With the available data, it is challenging to estimate any city-wide spatial effect due to the sparsity of the measured neighborhoods. However, within-neighborhood spatial effects could be feasible to estimate and could likely improve the model fit. 


\section{References}

Berghauser Pont, M. and P. Haupt (2010). Spacematrix. Space, density and urban form. Rotterdam: NAi Publishers.

Berghauser Pont, M. and L. Marcus (2015). What can typology explain that configuration cannot? In Proceedings of the 10th International Space Syntax Symposium. UCL, London.

Berghauser Pont, M., G. Stavroulaki, E. Bobkova, J. Gil, L. Marcus, J. Olsson, K. Sun, M. Serra, B. Hausleitner, A. Dhanani, and A. Legeby (2019). The spatial distribution and frequency of street, plot and building types across five european cities. Environment and Planning B: Urban Analytics and City Science 46(7), 1226-1242.

Berghauser Pont, M., G. Stavroulaki, and L. Marcus (2019). Development of urban types based on network centrality, built density and their impact on pedestrian movement. Environment and Planning B: Urban analytics and city science 46(8), 1549-1564.

Bolin, D., P. Guttorp, A. Januzzi, D. Jones, M. Novak, H. Podschwit, L. Richardson, A. Särkkä, C. Sowder, and A. Zimmerman (2015). Statistical prediction of global sea level from global temperature. Statist. Sinica 25(1), 351-367.

Bolin, D. and F. Lindgren (2015). Excursion and contour uncertainty regions for latent gaussian models. Journal of the Royal Statistical Society, Series B (Statistical Methodology) 77(1), 85-106.

Bolin, D. and F. Lindgren (2018). Calculating probabilistic excursion sets and related quantities using excursions. Journal of Statistical Software 86(5), 1-20.

Bolin, D. and J. Wallin (2020). Scale dependence: Why the average CRPS often is inappropriate for ranking probabilistic forecasts. Preprint, arXiv:1912.05642.

Chang, W., J. Cheng, J. Allaire, Y. Xie, and J. McPherson (2019). shiny: Web Application Framework for $R$. R package version 1.3.2.

Friedman, J. H. (2001). Greedy function approximation: A gradient boosting machine. The Annals of Statistics 29(5), 1189-1232.

Gneiting, T. and A. E. Raftery (2007). Strictly proper scoring rules, prediction, and estimation. Journal of the American Statistical Association 102(477), 359-378.

Hastie, T., R. Tibshirani, and J. H. Friedman (2009). The Elements of Statistical Learning: Data Mining, Inference, and Prediction. Springer.

Hillier, B., R. Burdett, J. Peponis, and A. Penn (1987). Creating life: or, does architecture determine anything? Architecture and Comportement/Architecture and Behaviour 3(3), 233-250.

Hillier, B. and S. Iida (2005). Network and psychological effects in urban movement. In Procceedings of the 5th International Space Syntax Symposium. Delft University of Technology. 
Hillier, B., A. Penn, J. Hanson, T. Grajewski, and J. Xu (1993). Natural movement: or, configuration and attraction in urban pedestrian movement. Environment and Planning B: Planning and Design 20, 29-66.

Hillier, B., T. Yang, and A. Turner (2012). Normalising least angle choice in depthmap and how it opens new perspectives on the global and local analysis of city space. Journal of Space Syntax 3, 155-193.

Hinton, G. E. (1990). Connectionist learning procedures. In Y. Kodratoff and R. S. Michalski (Eds.), Machine Learning, pp. 555 - 610. San Francisco (CA): Morgan Kaufmann.

Ho, T. K. (1995). Random decision forests. In Proceedings of the Third International Conference on Document Analysis and Recognition, ICDAR '95, pp. 278-282.

Kaufman, C. G. and S. R. Sain (2010). Bayesian functional ANOVA modeling using Gaussian process prior distributions. Bayesian Analysis 5(1), 123-149.

Legeby, A. (2013). Patterns of co-presence. Spatial configuration and social segregation. Stockholm: KTH University.

Netto, V., R. Sabayo, J. Vargas, L. Figueiredo, C. Freitas, and P. M. (2012). The convergence of patterns in the city: (isolating) the effects of architectural morphology on movement and activity. In Proceedings of the 8th International Space Syntax Symposium. Santiago de Chile: PUC.

Ozbil, A., J. Peponis, and B. Stone (2011). Understanding the link between street connectivity, land use and pedestrian flows. Urban Design International 16, 125-141.

Ozbil, A., D. Yesiltepe, and G. Argin (2015). Modeling walkability: the effects of street design, street-network configuration and land-use on pedestrian movement. A/Z ITU Journal of Faculty of Architecture 12, 189-207.

Pedregosa, F., G. Varoquaux, A. Gramfort, V. Michel, B. Thirion, O. Grisel, M. Blondel, P. Prettenhofer, R. Weiss, V. Dubourg, J. Vanderplas, A. Passos, D. Cournapeau, M. Brucher, M. Perrot, and E. Duchesnay (2011). Scikit-learn: Machine learning in Python. Journal of Machine Learning Research 12, 2825-2830.

Peponis, J., C. Feng, D. Green, D. Haynie, S. H. Kim, Q. Sheng, A. Vialard, and W. Haofeng (2015). Syntax and parametric analysis of superblock patterns. Journal of Space Syntax 6, 109-141.

Peponis, J., E. Hadjinikolaou, C. Livieratos, and D. A Fatouros (1989). The spatial core of urban culture. Ekistics 56, 43-55.

Peponis, J., C. Ross, and M. Rashid (1997). The structure of urban space, movement and co-presence: The case of atlanta. Geoforum 28(3-4), 341-358.

R Core Team (2018). R: A Language and Environment for Statistical Computing. Vienna, Austria: R Foundation for Statistical Computing. 
Read, S. (1999). Space syntax and the dutch city. Environment and Planning B: Planning and Design 26, 251-264.

Rue, H. and L. Held (2005). Gaussian Markov Random Fields. Chapman and Hall/CRC.

Rue, H., S. Martino, and N. Chopin (2009). Approximate Bayesian inference for latent Gaussian models by using integrated nested Laplace approximations. Journal of the Royal Statistical Society. Series B (Statistical Methodology) 71(2), 319-392.

Serra, M. and B. Hillier (2019). Angular and metric distance in road network analysis: A nationwide correlation study. Computers Environment and Urban Systems 74, 194-207.

Simpson, D., H. Rue, A. Riebler, T. G. Martins, and S. H. Sørbye (2017a). Penalising model component complexity: A principled, practical approach to constructing priors. Statistical Science 32(1), 1-28.

Simpson, D., H. Rue, A. Riebler, T. G. Martins, and S. H. Sørbye (2017b). You just keep on pushing my love over the borderline: A rejoinder. Statistical Science 32(1), 44-46.

Ståhle, A., L. Marcus, and A. Karlström (2017). Place syntax - geographic accessibility with axial lines in gis. Environment and Planning B: Planning and Design 26, 131-144.

Stavroulaki, G., D. Bolin, M. Berghauser Pont, L. Marcus, and E. Håkansson (2019). Statistical modelling and analysis of big data on pedestrian movement. In Proceedings of the 12th International Space Syntax Symposium, pp. 79.1-79-24. Beijing Jiaotong University.

Steadman, P. (2013). Density and built form: integrating spacemate with the work of martin and march. Environment and Planning B: Planning and Design 40, 341-358.

Wang, X., J. Liono, W. McIntosh, and F. D. Salim (2017). Predicting the city foot traffic with pedestrian sensor data. In Proceedings of the 14th EAI International Conference on Mobile and Ubiquitous Systems: Computing, Networking and Services, MobiQuitous 2017, New York, NY, USA, pp. 1-10. Association for Computing Machinery.

Yue, Y. R., D. Bolin, H. Rue, and X.-F. Wang (2019). Bayesian generalized two-way anova modeling for functional data using inla. Statistica Sinica 29, 741-767.

Zheng, Y., L. Capra, O. Wolfson, and H. Yang (2014). Urban computing: Concepts, methodologies, and applications. ACM Transactions on Intelligent Systems and Technology $5(3)$. 